\title{
The usefulness of laparoscopy-assisted distal gastrectomy in comparison with that of open distal gastrectomy for early gastric cancer
}

\author{
Hiroshi Yano ${ }^{1}$, Takushi Monden ${ }^{1}$, Masakatsu Kinuta ${ }^{1}$, Yoshiaki Nakano ${ }^{1}$, Takeshi Tono ${ }^{1}$, Shigeo Matsui ${ }^{1}$, \\ TAKashi Iwazawa ${ }^{1}$, ToshiYuki KanOH ${ }^{1}$, and ShinjI Katsushima ${ }^{2}$ \\ ${ }^{1}$ Department of Surgery, NTT West Osaka Hospital, 2-6-40, Karasugatsuji, Tennoji-ku, Osaka 543-8922, Japan \\ ${ }^{2}$ First Department of Internal Medicine, NTT West Osaka Hospital, Osaka, Japan
}

\begin{abstract}
Background. The technique of laparoscopy-assisted distal gastrectomy (LADG) was developed for early gastric cancer, but its feasibility and the associated clinical outcome remain unclear.

Methods. We reviewed 24 patients who underwent LADG (LADG group) and 35 patients who underwent traditional open distal gastrectomy (ODG group) for early gastric cancer in our hospital, and compared the clinical data of the two groups.

Results. The clinical and pathological backgrounds of the patients in the two groups were similar. The duration of surgery was not significantly different between the two groups, but the blood loss in the LADG group was significantly less than that in the ODG group. The number of removed lymph nodes was not significantly different between the two groups. The times to the first passing of flatus, first walking, and the restarting of oral intake; the length of hospital stay; and the duration of epidural analgesia were significantly shorter in the LADG group. The morbidity rate in the LADG group was lower than that in the ODG group.

Conclusions. LADG is a safe and minimally invasive surgical technique, after which we can expect a faster recovery.
\end{abstract}

Key words Laparoscopy-assisted distal gastrectomy · Gastric cancer - Minimally invasive surgery $\cdot$ Morbidity - Mortality · Outcome

\section{Introduction}

New techniques, such as endoscopic mucosal resection (EMR) [1-4], laparoscopic partial resection [5], and intragastric surgery [6], have been reported as minimally invasive surgery for early gastric cancer. However, in these operations, the regional lymph nodes (LNs) around the primary tumor cannot be resected.

Offprint requests to: $\mathrm{H}$. Yano

Received: February 23, 2001 / Accepted: June 19, 2001
Generally, LN metastases are seen in a small percentage of patients with early gastric cancer with mucosal or submucosal invasion [7]. In recent years, the technique of laparoscopy-assisted distal gastrectomy (LADG) with regional LN dissection has been developed and employed for early gastric cancer [8]. In March, 1997, we began to perform LADG as a minimally invasive surgery for early gastric cancer. However, the feasibility of LADG for early gastric cancer and the associated clinical outcome of patients who undergo LADG for early gastric cancer remain unclear.

We therefore conducted a review of patients who underwent LADG for early gastric cancer, in an effort to compare the operative times, intra-operative blood loss, number of removed lymph nodes, postoperative recovery, and morbidity and mortality rates of LADG and conventional open distal gastrectomy (ODG). Our research was aimed at determining whether the laparoscopic procedure of LADG for early gastric cancer is really safe and minimally invasive, and whether or not the LADG improves quality of life, compared with ODG.

\section{Patients and methods}

\section{Patients}

The patients were preoperatively diagnosed as having an early gastric cancer located in the lower or middle third of the stomach, from the results of endoscopy, endoscopic ultrasonography (EUS), and examination of biopsy specimens. The indications for LADG were that: (1) the tumor was located in the middle or lower part of the stomach, (2) the invasion of the tumor was limited to the mucosal layer or the submucosal layers (SM1). Tumors with deep submucosal invasion (SM2) were excluded from the indications for LADG. Between March 1997 and August 2000, LADG was performed 
in 24 patients with early gastric cancer at the Department of Surgery, NTT West Osaka Hospital. Another 35 patients, who underwent ODG with D1+ $\alpha$ lymph node dissection with mucosal or submucosal (SM1) invasion for early gastric cancer between January 1995 and December 1996, were also evaluated, as a control group. This study was carried out retrospectively, and we compared two populations operated at different time periods. No attempt was made to select the patients according to tumor size, macroscopic type, histologic type, or medical condition. The patients in both groups underwent surgery under general and epidural anesthesia. After surgery, the epidural tube was used for pain control and this was maintained until the patients no longer complained of abdominal pain. The same antibiotics were given, by injection, to the patients in both groups, for 5 days after surgery.

\section{Procedure for laparoscopy-assisted distal gastrectomy}

After pneumoperitoneum was established by Hasson's method with carbon dioxide, laparoscopic procedures were performed via four trocars. The stomach, abdominal cavity, and liver were explored with laparoscopic visualization, and we confirmed, macroscopically and cytologically, there was no liver metastasis or peritoneal dissemination. The greater omentum was cut with laparoscopic coagulation shears, the roots of the right gastroepiploic vessels were exposed, and Kocher's mobilization was performed so that we were able to get proper traction of the duodenum. An upper abdominal incision for mini-laparotomy, 7-cm-long, was made at the subxiphoid site. After supra (no. 5) and infra (no. 6)pyloric LNs were removed, the duodenum was transected under direct vision. The antral part of the stomach was pulled out through the mini-laparotomy incision. The right paracardial (no. 1), lesser curvature (no. 3), and left gastric artery (no. 7) LNs were removed, and, finally, the distal two-thirds of the stomach was resected with a stapling device. After the distal gastrectomy, a Billroth I anastomosis was established by a hand-suturing technique. A Penrose drain was placed at Winslow's foramen, and the abdominal wall was closed in layers. This LADG procedure was a distal gastrectomy that included D1+ lymph node no. 1 and 7 dissection.

\section{Procedure for conventional open distal gastrectomy}

Distal gastrectomy with regional LN dissection was carried out through an upper midline laparotomy approximately $20-\mathrm{cm}$ long. The regional LNs around the primary tumor were dissected to the same extent as that in the LADG. A Billroth I reconstruction was performed in a layer-to-layer manner.
The operative times, blood loss during the operation, number of removed LNs, length of hospital stay, times until the first flatus was passed and oral intake was resumed, duration of epidural analgesia, and complications were evaluated in both groups. The blood loss during the operation was determined by weighing the aspirated blood and the gauze with blood. Oral intake was begun with a liquid diet. Pathological classification was based on the Japanese classification of gastric carcinoma [9].

\section{Statistics}

The means and the SDs of the collected data were calculated. Data values were statistically analyzed by Student's $t$-test, using the StatView software package (SAS Institute, Cary, NC, USA). All results were considered statistically significant at $P$ values of less than 0.05 .

\section{Results}

The clinical backgrounds of the patients are shown in Table 1 . In the LADG group, three patients had recurrence after EMR, two patients had multiple cancers, and three patients with cholelithiasis or gallbladder polyp underwent laparoscopic cholecystectomy at the same time. In the ODG group, two patients had recurrence after EMR, and two patients had liver cirrhosis. One patient had cholelithiasis and underwent cholecystectomy at the same time. The clinical backgrounds of the patients in the two groups were similar. The pathological findings in the patients with gastric carcinoma are shown in Table 2. We found no differences between the LADG and ODG groups in location of the tumor, macroscopic type, depth of tumor invasion, histological

Table 1. Clinical backgrounds of patients with gastric carcinoma

\begin{tabular}{lcc}
\hline & $\begin{array}{c}\text { LADG } \\
(n=24)\end{array}$ & $\begin{array}{c}\text { ODG } \\
(n=35)\end{array}$ \\
\hline Sex & & \\
$\quad$ Male & 13 & 21 \\
$\quad$ Female & 11 & 14 \\
Mean age (years) & $61.7 \pm 1.4$ & $58.0 \pm 1.8$ \\
Comments & 3 & \\
$\quad$ Recurrence after EMR & 2 & 0 \\
$\quad$ Multiple cancers & & \\
Past history & 0 & 2 \\
$\quad$ Liver cirrhosis & 2 & 0 \\
$\quad$ Cholelithiasis & 1 & 1 \\
$\quad$ Gallbladder polyp & 0 &
\end{tabular}

LADG, Laparoscopy-assisted distal gastrectomy; ODG, open distal gastrectomy; EMR, endoscopic mucosal resection 
Table 2. Pathological findings in the patients with gastric carcinoma $^{\mathrm{a}}$

\begin{tabular}{|c|c|c|}
\hline & $\begin{array}{l}\mathrm{LADG} \\
(n=24)\end{array}$ & $\begin{array}{c}\text { ODG } \\
(n=35)\end{array}$ \\
\hline \multicolumn{3}{|c|}{ Location of tumor } \\
\hline $\mathrm{L}$ & 9 & 13 \\
\hline $\mathrm{M}$ & 15 & 22 \\
\hline \multicolumn{3}{|c|}{ Macroscopic type } \\
\hline $0-\mathrm{I}$ & 1 & 0 \\
\hline 0-IIa & 2 & 5 \\
\hline 0-IIc & 21 & 30 \\
\hline \multicolumn{3}{|c|}{ Depth of tumor invasion } \\
\hline M & 16 & 22 \\
\hline SM & 8 & 13 \\
\hline \multicolumn{3}{|l|}{ Histological type } \\
\hline tub & 16 & 20 \\
\hline por & 5 & 0 \\
\hline sig & 3 & 15 \\
\hline \multicolumn{3}{|c|}{ Lymphatic invasion } \\
\hline lyo & 24 & 34 \\
\hline ly2 & 0 & 1 \\
\hline \multicolumn{3}{|l|}{ Venous invasion } \\
\hline v0 & 24 & 34 \\
\hline $\mathrm{v} 1$ & 0 & 1 \\
\hline \multicolumn{3}{|c|}{ Extent of lymph node metastasis } \\
\hline No & 24 & 34 \\
\hline $\mathrm{N} 1$ & 0 & 1 \\
\hline \multicolumn{3}{|l|}{ Stage grouping } \\
\hline IA & 24 & 34 \\
\hline IB & 0 & 1 \\
\hline \multicolumn{3}{|c|}{ Curative potential of gastric resection } \\
\hline Resection A & 24 & 35 \\
\hline \multicolumn{3}{|c|}{$\begin{array}{l}\text { L, Lower third; M, middle third; 0-I, protruded type; } 0 \text {-IIa, superficial } \\
\text { elevated type; 0-IIc, superficial depressed type; M, tumor invasion of } \\
\text { mucosa and/or muscularis mucosa; SM, tumor invasion of submucosa; } \\
\text { tub, tubular adenocarcinoma; por, poorly differentiated adenocarci- } \\
\text { noma; sig, signet-ring cell carcinoma; ly0, no lymphatic invasion; ly2, } \\
\text { moderate lymphatic invasion; v0, no venous invasion; v1, minimal } \\
\text { venous invasion; N0, no evidence of lymph node metastasis; N1, me- } \\
\text { tastasis to group } 1 \text { lymph nodes; resection A, no residual disease; high } \\
\text { probability of cure } \\
\text { a Pathological findings classified according to reference [9] }\end{array}$} \\
\hline
\end{tabular}

type, lymphatic invasion, venous invasion, extent of lymph node metastasis, stage grouping, and curative potential of gastric resection.

Details of the operative course and postoperative recovery were evaluated in the two groups (Table 3 ). The mean operative time was not significantly different between the two groups, but the mean intra-operative blood loss in the LADG group was significantly less than that in the ODG group $(P=0.0001)$. The times to the first passing of flatus $(P=0.01)$, the first walking $(P=0.0001)$, and the restarting of oral intake $(P=$ $0.01)$; the length of hospital stay $(P=0.01)$; and the duration of epidural analgesia $(P=0.0001)$ were all significantly shorter in the LADG group. The number of removed lymph nodes was not significantly different between the two groups (Table 4).

The morbidity and mortality rates in the LADG and ODG groups are shown in Table 5. In the LADG group, there was only one complication, a case of anastomotic stenosis, and there was no recurrence for 3 years. In the patient with anastomotic stenosis, this was diagnosed by Gastrografin (Schering, Osaka, Japan) contrast study on the seventh postoperative day. The condition resolved with conservative treatments, and the patient was discharged on the seventieth day after the surgery. The total morbidity and mortality rates in

Table 4. Number of removed lymph nodes

\begin{tabular}{lcc}
\hline LADG & ODG & $P$ \\
\hline $18.5 \pm 2.3$ & $23.9 \pm 1.8$ & NS \\
\hline
\end{tabular}

Table 5. Morbidity and mortality rates in the LADG and ODG groups

\begin{tabular}{lcl}
\hline & LADG $(n=24)$ & ODG $(n=35)$ \\
\hline Wound infection & 0 & $1(2.9 \%)$ \\
Ileus & 0 & $1(2.9 \%)$ \\
Anastomotic stenosis & $1(4.2 \%)$ & $1(2.9 \%)$ \\
Anastomotic leakage & 0 & $1(2.9 \%)$ \\
Total & $1(4.2 \%)$ & $4(11.4 \%)$ \\
Mortality & 0 & 0 \\
\hline
\end{tabular}

Table 3. Details of operative course and postoperative recovery

\begin{tabular}{lccc}
\hline & LADG & ODG & $P$ \\
\hline Mean operative time (min) & $219.8 \pm 9.4$ & $210.0 \pm 8.6$ & $\mathrm{NS}$ \\
Mean intra-operative blood loss (g) & $108.4 \pm 16.3$ & $296.1 \pm 35.9$ & 0.0001 \\
Time to first flatus (days) & $2.71 \pm 0.25$ & $3.56 \pm 0.20$ & 0.01 \\
Time to first walking (days) & $1.25 \pm 0.09$ & $2.30 \pm 0.17$ & 0.0001 \\
Time to recommencement of oral intake & $4.54 \pm 0.32$ & $5.56 \pm 0.24$ & 0.01 \\
$\quad$ (days) & $20.9 \pm 2.4$ & $29.4 \pm 2.1$ & 0.01 \\
Length of hospital stay (days) & $2.74 \pm 0.21$ & $5.00 \pm 0.29$ & 0.0001 \\
Duration of epidural analgesia (days) & & &
\end{tabular}

NS, Not significant 
the LADG group were $4.2 \%$ and $0 \%$, respectively. On the other hand, in the ODG group, we experienced minor complications, with one case of wound infection, one of postoperative ileus, one of anastomotic stenosis, and one of anastomotic leakage. The total morbidity and mortality rates in the ODG group were $11.4 \%$ and $0 \%$, respectively.

\section{Discussion}

Early gastric cancer confined to the mucosa or submucosa can be diagnosed with increased accuracy by endoscopy and endoscopic ultrasonography (EUS) [10,11]. A variety of minimally invasive treatments for early gastric cancer have been reported, such as EMR [1-4], laparoscopic wedge resection [5], and laparoscopic intraluminal surgery [6]. However, these operations do not include regional LN dissection. The incidence of lymphatic metastasis is related to the depth of invasion of the primary tumor, and ranges from $4 \%$ for tumors confined to the mucosa to $20 \%$ for tumors extending to the submucosa [7]. Therefore, it would seem logical to carry out the resection of perigastric LNs, at least for patients with early gastric cancer. In this respect, LADG has higher curability (similar to that of conventional ODG with regional LN dissection) than that of the above-mentioned operations [1-6]. However, the usefulness of and indications for LADG still remain unclear. To elucidate this question, we compared the clinical data of LADG and ODG groups to determine whether LADG is really minimally invasive and whether it enhances quality of life.

The times to the first passing of flatus, the first walking, and the restarting of oral intake, as well as the length of hospital stay and duration of epidural analgesia, were significantly shorter in the LADG group than in the ODG group. All these results suggest that LADG is a minimally invasive technique and that it improves the quality of life, compared with ODG.

The mean operative time was not significantly different between the two groups. The mean intra-operative blood loss in the LADG group was significantly less than that in the ODG group. The number of removed LNs was not significantly different between the two groups. In the LADG group, there was only one complication (a case of anastomotic stenosis), and there was no recurrence for 3 years. The total morbidity and mortality rates in the LADG group were $4.2 \%$ and $0 \%$, respectively, while, in contrast, the total morbidity and mortality rates in the ODG group were $11.4 \%$ and $0 \%$, respectively. All these results indicate that LADG is a safe procedure with high curability.

The advantage of LADG, compared with EMR, a lesion-lifting method, or intragastric surgery, is that re- gional LNs can be removed. Stage grouping and surgical curability can be histologically evaluated because of the removal of regional LNs around the primary tumor. If intra-operative pathological diagnosis shows that the resected LNs include many metastatic lesions, we can convert to open surgery with widely extended LN dissection, because it is extremely difficult to completely remove the LNs along the common hepatic, splenic, or celiac arteries by the LADG procedure. For the purpose of removing regional LNs completely, we did it under direct vision through a small incisional wound, as in open surgery. Recently, a completely laparoscopic approach for the removal of regional LNs was reported, but the procedure involved a very difficult technique and a long operation time [12].

The indications for LADG are limited to early gastric cancer, because wide LN dissection is generally needed for advanced gastric cancer. We excluded tumors with deep submucosal invasion (SM2) from the indications for LADG, because these tumors were reported to be associated with level 2 lymph node metastasis [13]. However, tumors with mucosal or slight submucosal invasion (SM1) were completely resected, with high curability, by LADG. EUS was useful for the preoperative detection of the depth of invasion of gastric cancers. The accuracy of EUS for determining depth of invasion of early gastric cancers was reported to be approximately $70 \%$ [10-11]. Another advantage of LADG, compared with EMR, is that the tumor can be completely removed with a wide surgical margin, by the resection of the distal two-thirds of the stomach. However, only cancerous lesions of the middle or lower parts of the stomach can be resected by LADG. Total or proximal gastrectomy is the procedure of choice for gastric cancer located in the upper part of the stomach. After resection for cancer in the upper part of the stomach, esophago-jejunostomy is too difficult to perform safely by a laparoscopic technique.

In conclusion, LADG is a safe and minimally invasive form of surgery from which faster recovery can be expected than from ODG. The indication for LADG is limited to early gastric cancer located in the middle or lower parts of stomach. Only a few parameters in the early postoperative course were investigated in our study. Further investigations of the late postoperative course will be necessary, including comparisons of prognosis, recurrent rate, and complications.

\section{References}

1. Hiki Y. Endoscopic treatment for gastric cancer from the surgical perspective. Gastroenterol Endosc 1991;33:2285-99.

2. Lambert R. Endoscopic therapy of esophago-gastric tumors. Endoscopy 1992;24:24-33.

3. Tada M, Murakami A, Karita M, Yanai H, Okita K. Endoscopic resection of early gastric cancer. Endoscopy 1993;25:445-50. 
4. Takekoshi T, Baba Y, Ota H, Kato Y, Yanagisawa A, Takagi K, Noguchi Y. Endoscopic resection of early gastric carcinoma: results of a retrospective analysis of 308 cases. Endoscopy 1994; 26:352-8.

5. Ohgami M, Kumai K, Otani Y, Wakabayashi G, Kubota T, Kitajima M. Laparoscopic wedge resection of the stomach for early gastric cancer using a lesion-lifting method. Dig Surg 1994; 11:64-7.

6. Ohashi S. Laparoscopic intraluminal (intragastric) surgery for early gastric cancer. A new concept in laparoscopic surgery. Surg Endosc 1995;9:169-71.

7. Fukutomi H, Sakita T. Analysis of early gastric cancer cases collected from major hospitals and institutes in Japan. Jpn J Clin Oncol 1984;14:169-79.

8. Kitano S, Iso Y, Moriyama M, Sugimachi K. Laparoscopy-assisted Billroth I gastrectomy. Surg Laparosc Endosc 1994;4:146-8.

9. Japanese Gastric Cancer Association. Japanese classification of gastric carcinoma 13th ed (in Japanese). Tokyo: Kanehara; 1999.
10. Akahoshi K, Chijiiwa Y, Hamada S, Sasaki I, Nawata H, Kabemura T, et al. Pretreatment staging of endoscopically early gastric cancer with a $15 \mathrm{MHz}$ ultrasound catheter probe. Gastrointest Endosc 1998;48:470-6.

11. Wang JY, Hsieh JS, Huang YS, Huang CJ, Hou MF, Huang TJ. Endoscopic ultrasonography for preoperative locoregional staging and assessment of resectability in gastric cancer. Clin Imaging 1998;22:355-9.

12. Uyama I, Sugioka A, Fujita J, Komori Y, Matsui H, Soga R, et al. Completely laparoscopic extraperigastric lymph nodes dissection for gastric malignancies located in the middle or lower third of the stomach. Gastric Cancer 1999;2:186-90.

13. Shiraishi N, Adachi Y, Kitano S, Bandoh T, Katsuta T, Morimoto A. Indication for and outcome of laparoscopy-assisted Billroth I gastrectomy. Br J Surg 1999;86:541-4. 